\title{
FUZZY TOPOLOGICAL DIGITAL SPACE OF FLAT ELECTROENCEPHALOGRAPHY DURING EPILEPTIC SEIZURES
}

\author{
Muhammad Abdy and Tahir Ahmad \\ Department of Mathematics, Nanotechnology Research Alliance, \\ Theoretical and Computational Modeling for Complex Systems, \\ Universiti Teknologi Malaysia, 81310 Skudai, Johor Malaysia
}

Received 2013-05-21, Revised 2013-06-21; Accepted 2013-07-05

\begin{abstract}
Epileptic seizures are manifestations of epilepsy caused a temporary electrical disturbance in a group of brain cells. Electroencephalography (EEG) is a recording of electrical activity of the brain and it contains valuable information related to the different physiological states of the brain. Flat EEG (fEEG) was developed to compress and analyze information in the brain during epileptic seizures obtained from EEG signal. In this study, the $f E E G$ is made in digital form by using Voronoi digitization. Khalimsky fuzzy topology and fuzzy topological digital space is constructed to be used in studying properties fuzzy topology of $f$ EEG. It is obtained that $f$ EEG is a fuzzy topological digital space and $\tau$-connected.
\end{abstract}

Keywords: Flat EEG, Fuzzy Topological Digital Space, Khalimsky Fuzzy Topology

\section{INTRODUCTION}

Epileptic seizures are manifestations of epilepsy caused a temporary electrical disturbance in a group of brain cells (neurons). The recording of electrical signals emanated from the human brain, which can be collected from the scalp of the head is called Electroencephalography (EEG). Careful analysis of the EEG records can provide new insights into the epileptogenic process and may have considerable utilization in the diagnosis and treatment of epilepsy (Tahir, 2010).

The methods in information geometry were developed by Amari and Nagaoka (2000) as early since 1993. It has gain a big momentum when dealing with statistical data. On the other hand, (Zakaria, 2008) has developed a novel method to map high dimensional signal, namely EEG signal into low dimensional space (MC). The process is a transformation of EEG signals originating from the patient's head into two dimensional planes. The result of the transformation of the EEG signals into low-dimensional space is called as flat EEG ( $f$ EEG). $f$ EEG has been used purely for visualization, but the main scientific value will lie in the ability of flattening method to preserve information. The 'jewel' of the $f$ EEG method is EEG signals can be compressed and analyzed.

Nazihah et al. (2009) have constructed $f$ EEG as a digital space. They proved that the digital space of the $f$ EEG is an Alexsandroff space and have applied relational topology in order to incorporate topological space of real time recorded EEG signal with digital $f$ EEG. This construction is a key foundation of $f$ EEG which has linked with the idea of fuzzy information granulation. All points in digital $f E E G$ which give the coordinate of the location of the current source are supposed to be in the structure of discrete objects. It means that each of the points possesses smallest neighborhood in analogy of digital topology. It can be a point or a small portion of region which contains several points that caused the dysfunction of brain. Thus, every

\footnotetext{
Corresponding Author: Tahir Ahmad, Department of Mathematics, Nanotechnology Research Alliance,

Theoretical and Computational Modeling for Complex Systems, Universiti Teknologi Malaysia,

81310 Skudai, Johor Malaysia
} 
point is said to be integer grid on the plane. These points can also carry information such as magnetic field and images (Nazihah, 2009)

\section{MATERIALS AND METHODS}

\subsection{Flat EEG Digital}

$f$ EEG is digitized into grid by using Voronoi digitization (Kiselman, 2004), as follows:

Let $\mathrm{x} \in \mathrm{R}, \mathrm{p} \in \mathrm{Z}$ then the Voronoi cell with nucleus $\mathrm{p}$ is Equation (1):

$$
\operatorname{Vo}(p)=\{x \in \mathbb{R} \mid \forall q \in \mathbb{Z}, d(x, p) \leq d(x, q)\}
$$

On the $x$-axis of the $f$ EEG, the Voronoi cell is Equation (2):

$$
\operatorname{Vo}(p)=\left\{x \in \mathbb{R} \mid p-\frac{1}{2}<x \leq p+\frac{1}{2}, p \in \mathbb{Z}\right\}
$$

Each $\mathrm{x} \in \mathrm{R}$ is a member in only one Voronoi cell $\operatorname{Vo}(p)$ for every $\mathrm{p} \in \mathrm{Z}$, so that the $x$-axis Voronoi digitization of the $f$ EEG has the following form Equation (3):

$$
\operatorname{dig}[\{x\}]=\{p \in \mathbb{Z} \mid x \in \operatorname{Vo}(p)\}
$$

$f$ EEG is the Cartesian product of the $\mathrm{x}$-axis and $\mathrm{y}$ axis, so that the digitization of the $f$ EEG is the Cartesian product of the digitization of the $\mathrm{x}$-axis and y-axis, namely Equation (4):

$$
\operatorname{dig}[\{(\mathrm{x}, \mathrm{y})\}]=\left\{\left(\mathrm{p}_{\mathrm{x}}, \mathrm{p}_{\mathrm{y}}\right) \in \mathbb{Z}^{2} \mid \mathrm{x} \in \operatorname{Vo}\left(\mathrm{p}_{\mathrm{x}}\right), \mathrm{y} \in \operatorname{Vo}\left(\mathrm{p}_{\mathrm{y}}\right)\right\}
$$

Where:

$$
\operatorname{Vo}\left(p_{x}\right)=\left\{x \in \mathbb{R} \mid p_{x}-\frac{1}{2}<x \leq p_{x}+\frac{1}{2}, p_{x} \in \mathbb{Z}\right\}
$$

And:

$$
\operatorname{Vo}\left(p_{y}\right)=\left\{y \in \mathbb{R} \mid p_{y}-\frac{1}{2}<y \leq p_{y}+\frac{1}{2}, p_{y} \in \mathbb{Z}\right\}
$$

Hence, the $f$ EEG in digital form Equation (5):

$$
\begin{aligned}
& \mathrm{fEEG}=\left\{(\mathrm{p}, \text { Volt }) \mid \mathrm{p}=\left(\mathrm{p}_{\mathrm{x}}, \mathrm{p}_{\mathrm{y}}\right) \in \mathbb{Z}^{2} ; \text { Volt } \in \mathbb{R}^{+}\right\} \\
& =\left\{(\mathrm{x}, \mathrm{y})_{\text {volt }} \mid \mathrm{x}, \mathrm{y} \in \mathbb{Z} ; \text { Volt } \in \mathbb{R}^{+}\right\}
\end{aligned}
$$

With volt is electric potential.

\subsection{Alexandroff Fuzzy Topological Space}

Alexandroff topological space (Kopperman, 2003) is extended to fuzzy topological space and presented formally as follows.

\section{Definition 1}

Let $(X, \tilde{\tau})$ be a fuzzy topological space, then $(X, \tilde{\tau})$ is called Alexandroff fuzzy topological space if the intersection of any open fuzzy set in $\mathrm{X}$ is open, i.e.:

$$
\text { If } \tilde{\mathrm{A}}_{\mathrm{i}} \in \tilde{\tau} \text { then }\left(\bigcap_{\mathrm{i}} \tilde{\mathrm{A}}_{\mathrm{i}}\right) \in \tilde{\tau} \text {, for }\left\{\tilde{\mathrm{A}}_{\mathrm{i}} \mid \mathrm{i} \in \mathrm{I}\right\}
$$

The smallest neighborhood (Kiselman, 2004) is extended to fuzzy topology on X.

\section{Definition 2}

Let $(\mathrm{X}, \tilde{\tau})$ be a fuzzy topological space. The smallest neighborhood of $c \in X$ (with respect to $\tilde{\tau}$ ) is defined by:

$$
\mathrm{S}_{\tilde{\tau}}(\mathrm{c})=\bigcap_{\mathrm{c} \in \tilde{U} \in \tilde{\tau}} \tilde{U}=\min _{\tilde{U} \in \tilde{\tau}}\left(\mu_{\tilde{U}}(\mathrm{c})\right)
$$

\section{Theorem 1}

Let $(X, \tilde{\tau})$ be a fuzzy topological space, then $(X, \tilde{\tau})$ is an Alexandroff fuzzy topological space if and only if $\forall \mathrm{x} \in \mathrm{X}, x$ possesses the smallest open neighborhood, i.e., $\forall \mathrm{x} \in \mathrm{X}, \tilde{\mathrm{S}}_{\tilde{\tau}}(\mathrm{x})=\bigcap_{\mathrm{x} \in \tilde{\mathrm{U}} \in \tilde{\tau}} \tilde{\mathrm{U}}$ is an open fuzzy set in $\mathrm{X}$.

\section{Proof}

Suppose $(\mathrm{X}, \tilde{\tau})$ is an Alexandroff fuzzy topological space with $\mathrm{x} \in \mathrm{X}$. Consider $\mathrm{N}(\mathrm{x})=$ $\{\tilde{\mathrm{N}} \subset \mathrm{X} \mid \tilde{\mathrm{N}}$ is an open neighborhood of $\mathrm{x}\}$. Pick $\tilde{\mathrm{S}}(\mathrm{x})=\bigcap \tilde{\mathrm{N}}$ for $\tilde{\mathrm{N}} \in \mathrm{N}(\mathrm{x})$, then $\tilde{\mathrm{S}}(\mathrm{x})$ is an open fuzzy set because $X$ is an Alexandroff fuzzy topological space. Hence, $\tilde{S}(x)$ is an open neighborhood of $\mathrm{x}$. Based on the intersection definition, it is clear that $\tilde{S}(x)$ is a smallest open neighborhood of $\mathrm{x}$. 
Suppose $\forall \mathrm{x} \in \mathrm{X}, \quad x$ has the smallest open neighborhood $\tilde{\mathrm{S}}(\mathrm{x})$. Let $\tilde{\mathrm{V}}=\bigcap_{\mathrm{U}_{\mathrm{i}}}$ be an arbitrary intersection of open set, where, $\tilde{U}_{\mathrm{i}}$ is open in X. If $\tilde{\mathrm{V}}=\varnothing$ then $\mathrm{V}$ is open and we are done. If $\tilde{\mathrm{V}} \neq \varnothing$ and pick $\mathrm{x} \in \tilde{\mathrm{V}}$, then $\mathrm{x} \in \tilde{\mathrm{U}}_{\mathrm{i}}, \forall \mathrm{i} \in \mathrm{I}$ so that $\tilde{\mathrm{S}}(\mathrm{x}) \subseteq \tilde{\mathrm{U}}_{\mathrm{i}}, \forall \mathrm{i} \in \mathrm{I}$ because $\tilde{\mathrm{S}}(\mathrm{x})$ is the smallest open fuzzy set containing $x$. Therefore, $\tilde{\mathrm{S}}(\mathrm{x}) \subseteq \tilde{\mathrm{V}}$. Hence, $\tilde{\mathrm{V}}$ is open because it contains an open set around each of its points.

\subsection{Khalimsky Fuzzy Topological on $Z$ and $Z^{2}$}

Khalimsky topology $\tau_{z}$ (Melin, 2008) is extended to fuzzy topology on Z. The construction of the fuzzy topological is done by a collection of fuzzy sets that is a base that will generate a fuzzy topology.

Let $\tilde{\mathrm{B}}(\mathrm{n})$ be a fuzzy set on $\mathrm{Z}$ as follows Equation (6):

$$
\begin{aligned}
& \tilde{\mathrm{B}}(\mathrm{n})= \\
& \left\{\begin{array}{l}
\left\{\left(\mathrm{n}, \mu_{\tilde{\mathrm{B}}(\mathrm{n})}(\mathrm{n})\right)\right\} \text { iif } \mathrm{n} \text { is odd } \\
\left\{\begin{array}{l}
\left(\mathrm{n}-1, \mu_{\tilde{\mathrm{B}}(\mathrm{n})}(\mathrm{n}-1)\right),\left(\mathrm{n}, \mu_{\tilde{\mathrm{B}}(\mathrm{n})}(\mathrm{n})\right), \\
\left(\mathrm{n}+1, \mu_{\tilde{\mathrm{B}}(\mathrm{n})}(\mathrm{n}+1)\right)
\end{array}\right\} \text { iif nis even }
\end{array}\right.
\end{aligned}
$$

Collection of the fuzzy sets $\tilde{B}(n), n \in Z$ is denoted by $\tilde{\beta}=\{\tilde{B}(n) \mid n \in \mathbb{Z}\}$ and collection of all possible union of elements of $\tilde{\beta}$ is denoted by $\tilde{\tau}=\{\cup \mathcal{Z} \mid \mathcal{Z} \subset \tilde{\beta}\}$

\section{Theorem 2}

$(\mathbb{Z}, \tilde{\tau})$ is a fuzzy topological space

\section{Proof}

We use definition of fuzzy topological space in (Srivastava et al., 1981):

- Pick $\mathcal{Z}=\varnothing$, then $\mathcal{Z}=\varnothing \subset \tilde{\beta}$, so that $\cup \varnothing=\varnothing$. Hence, $\varnothing \in \tilde{\tau}$. Therefore, $\tilde{\tau}$ contain constant fuzzy set

- Let $\tilde{\mathrm{A}}_{1}, \tilde{\mathrm{A}}_{2} \in \tilde{\tau}$ with $\tilde{\mathrm{A}}_{1}=\bigcup_{\mathrm{i}} \tilde{\mathrm{B}}\left(\mathrm{n}_{\mathrm{li}}\right), \tilde{\mathrm{A}}_{2}=\bigcup_{\mathrm{j}} \tilde{\mathrm{B}}\left(\mathrm{n}_{2 \mathrm{j}}\right)$ and $\tilde{\mathrm{B}}\left(\mathrm{n}_{1 \mathrm{i}}\right), \tilde{\mathrm{B}}\left(\mathrm{n}_{2 \mathrm{j}}\right) \in \tilde{\beta}$

$$
\begin{aligned}
& \left(\tilde{\mathrm{A}}_{1} \cap \tilde{\mathrm{A}}_{2}\right) \\
& =\left(\bigcup_{\mathrm{i}} \tilde{\mathrm{B}}\left(\mathrm{n}_{\mathrm{li}}\right)\right) \cap\left(\bigcup_{\mathrm{j}} \tilde{\mathrm{B}}\left(\mathrm{n}_{2 \mathrm{j}}\right)\right)=\bigcup_{\mathrm{i}, \mathrm{j}}\left(\left(\tilde{\mathrm{B}}\left(\mathrm{n}_{\mathrm{li}}\right)\right) \cap\left(\tilde{\mathrm{B}}\left(\mathrm{n}_{2 \mathrm{j}}\right)\right)\right)
\end{aligned}
$$

There are two possible results of finite intersection of element $\tilde{\beta}$, i.e.,

$\left(\tilde{B}\left(n_{1 \mathrm{i}}\right) \cap \tilde{B}\left(\mathrm{n}_{2 \mathrm{j}}\right)\right)=\varnothing$ or $\left(\tilde{\mathrm{B}}\left(\mathrm{n}_{1 \mathrm{i}}\right) \cap \tilde{\mathrm{B}}\left(\mathrm{n}_{2 \mathrm{j}}\right)\right) \in \tilde{\beta}$, so that $\left(\tilde{\mathrm{A}}_{1} \cap \tilde{\mathrm{A}}_{2}\right)=\bigcup \varnothing=\varnothing \in \tilde{\tau}$ or $\left(\tilde{\mathrm{A}}_{1} \cap \tilde{\mathrm{A}}_{2}\right)=\bigcup_{\mathrm{k}} \tilde{\mathrm{B}}\left(\mathrm{n}_{\mathrm{k}}\right)$ where $\tilde{\mathrm{B}}\left(\mathrm{n}_{\mathrm{k}}\right) \in \tilde{\beta}$. Hence, $\left(\tilde{\mathrm{A}}_{1} \cap \tilde{\mathrm{A}}_{2}\right) \in \tilde{\tau}$

- Let $\left\{\tilde{\mathrm{A}}_{\mathrm{i}}\right\}, \forall \mathrm{i} \in \mathrm{I} \quad$ where $\quad \tilde{\mathrm{A}}_{\mathrm{i}} \in \tilde{\tau}$, with $\tilde{\mathrm{A}}_{1}=\bigcup_{\mathrm{j}} \tilde{\mathrm{B}}\left(\mathrm{n}_{1 \mathrm{j}}\right), \tilde{\mathrm{A}}_{2}=\bigcup_{\mathrm{j}} \tilde{\mathrm{B}}\left(\mathrm{n}_{2 \mathrm{j}}\right), \ldots ; \quad \tilde{\mathrm{B}}\left(\mathrm{n}_{\mathrm{ij}}\right) \in \tilde{\beta}, \bigcup_{\mathrm{i}} \tilde{\mathrm{A}}_{\mathrm{i}}=$ $\tilde{A}_{1} \cup \tilde{A}_{2} \cup \ldots=\left(\bigcup_{j} \tilde{B}\left(n_{1 j}\right)\right) \cup\left(\bigcup_{j} \tilde{B}\left(n_{2 j}\right)\right) \cup \ldots=$ $\bigcup_{i}\left(\bigcup_{j} \tilde{B}\left(n_{i j}\right)\right)=\bigcup_{i, j} \tilde{B}\left(n_{i j}\right)$

Therefore, $\bigcup_{i} \tilde{A}_{i}$ can be expressed as union of element $\tilde{\beta}$. Hence, $\bigcup \tilde{A}_{i} \in \tilde{\tau}$.

Fuzzy topology $\tilde{\tau}$ in Theorem 2 is called as Khalimsky fuzzy topology, denoted by $\tilde{\tau}_{\mathbb{Z}}$ and $\left(\mathbb{Z}, \tilde{\tau}_{\mathbb{Z}}\right)$ is called Khalimsky fuzzy topological space. $\mathrm{Z}$ together with $\tilde{\tau}_{\mathbb{Z}}$ is called as Khalimsky fuzzy line.

\section{Theorem 3}

$\left(\mathbb{Z}^{22}, \tilde{\tau}_{\mathbb{Z}^{2}}\right)$ is a fuzzy topological space

\section{Proof}

Since $\left(\mathbb{Z}, \tilde{\tau}_{\mathbb{Z}}\right)$ is a fuzzy topological space then $\tilde{\tau}_{\mathbb{Z}^{22}}$ is a fuzzy topology generated by family $\left\{\mathrm{A}_{\mathrm{j}} \times \mathrm{B}_{\mathrm{k}} \mid \mathrm{A}_{\mathrm{j}}, \mathrm{B}_{\mathrm{k}} \in \tilde{\tau}_{\mathbb{Z}}\right\}$ (Palaniappan, 2005), so that $\left(\mathbb{Z}^{22}, \tilde{\tau}_{\mathbb{Z}^{22}}\right)$ is a fuzzy topological space.

The topology $\tilde{\tau}_{\mathbb{Z}^{2}}$ on $Z^{2}$ is called as Khalimsky fuzzy topology on $Z^{2}$ and $Z^{2}$ together with $\tilde{\tau}_{\mathbb{Z}^{2}}$ is called Khalimsky fuzzy plane.

The elements of the family $\left\{\mathrm{A}_{\mathrm{j}} \times \mathrm{B}_{\mathrm{k}} \mid \mathrm{A}_{\mathrm{j}}, \mathrm{B}_{\mathrm{k}} \in \tilde{\tau}_{\mathbb{Z}}\right\}$ as follows Equation (7): 


$$
\begin{aligned}
& \tilde{\mathrm{B}}(\mathrm{p})=
\end{aligned}
$$

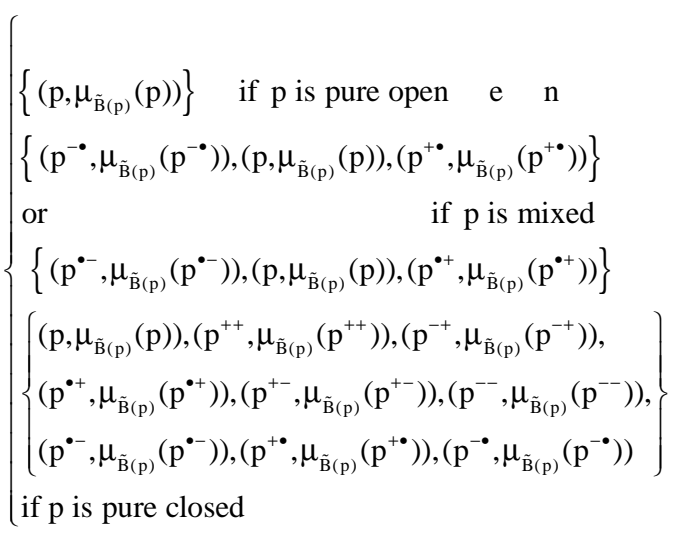

with $\mathrm{p}=(\mathrm{x}, \mathrm{y}) \in \mathrm{Z}^{2} ; \mathrm{p}^{++}=(\mathrm{x}+1, \mathrm{y}+1) ; \mathrm{p}^{-+}=(\mathrm{x}-1, \mathrm{y}+1) ; \mathrm{p}^{\bullet+}$ $=(\mathrm{x}, \mathrm{y}+1) ; \mathrm{p}^{+-}=(\mathrm{x}+1, \mathrm{y}-1) ; \mathrm{p}^{-}=(\mathrm{x}-1, \mathrm{y}-1) ; \mathrm{p}^{\bullet-}=(\mathrm{x}, \mathrm{y}-1) ;$ $\mathrm{p}^{+\bullet}=(\mathrm{x}+1, \mathrm{y}) ; \mathrm{p}^{-\bullet}=(\mathrm{x}-1, \mathrm{y})$.

Based on the definition 2, Theorem 1 and properties of $\tilde{B}(p)$, we have the following corollaries:

\section{Corollary 1}

$\tilde{\mathrm{B}}(\mathrm{p})$ is the smallest open neighborhood of $\mathrm{p}$.

\section{Corollary 2}

A Khalimsky fuzzy topological space $\left(\mathbb{Z}, \tilde{\tau}_{\mathbb{Z}}\right)$ is an Alexandroff fuzzy topological space.

On the Khalimsky fuzzy plane, $\tilde{\mathrm{B}}(\mathrm{p})$ is the smallest open neighborhood of $p$ on the plane (Corollary 1), so that $\tilde{\mathrm{B}}(\mathrm{p})$ define adjacency $\rho_{\tilde{\tau}_{z^{2}}}$ on $Z^{2}$ (Herman, 1998), i.e., $\forall \mathrm{p}, \mathrm{q} \in \mathrm{Z}^{2}$ then $(\mathrm{p}, \mathrm{q}) \in \rho_{\tilde{\tau}_{\mathrm{Z}^{2}}}$ if and only if $\mathrm{p} \neq \mathrm{q}$ and $\mathrm{p} \in \tilde{\mathrm{B}}(\mathrm{q})$ or $q \in \tilde{B}(p)$. The adjacency induced by Khalimsky fuzzy topology $\tilde{\tau}_{\mathbb{Z}^{2}}$ is called Khalimsky adjacency.

\subsection{Fuzzy Topological Digital Space}

Topological digital space (Herman, 1998) is extended to fuzzy topology and it is called fuzzy topological digital space

\section{Definition 3}

Let $\mathrm{V}$ be any set and $\pi$ is an adjacency on V. A digital space $(V, \pi)$ is called fuzzy topological digital space if there exist a fuzzy topology $\tilde{\tau}$ on $\mathrm{V}$, such that for any fuzzy set $\tilde{\mathrm{A}}$ in $\mathrm{V}$, if $\tilde{\mathrm{A}}$ is $\pi$-connected then $\tilde{\mathrm{A}}$ is $\tilde{\tau}$ - connected (topologically connected)

\section{Definition 4}

Let $(X, \tilde{\tau})$ be a fuzzy topological space. The adjacency induced by fuzzy topology $\tilde{\tau}$, denoted $\rho_{\tilde{\tau}}$, is defined as: $(c, d) \in \rho_{\tilde{\tau}}$ if and only if $c \neq d$ and $\mathrm{c} \in \mathrm{S}_{\tilde{\tau}}(\mathrm{d})$ or $\mathrm{d} \in \mathrm{S}_{\tilde{\tau}}(\mathrm{c}), \forall \mathrm{c}, \mathrm{d} \in \mathrm{X}$.

\section{Theorem 4}

Let $(X, \tilde{\tau})$ be an Alexandroff fuzzy topological space and $\tilde{\mathrm{A}}$ is any fuzzy set on $X$, then $\tilde{\mathrm{A}}$ is $\rho_{\tilde{\tau}}$ - connected if and only if $\tilde{A}$ is $\tilde{\tau}$ - connected.

\section{Proof}

We prove it from the left, the rest is left.

Now we show that if there exist $\tilde{U}, \tilde{V} \in \tilde{\tau}$ such that $\tilde{\mathrm{U}} \cap \tilde{\mathrm{A}} \neq \varnothing, \quad \tilde{\mathrm{V}} \cap \tilde{\mathrm{A}} \neq \varnothing \quad$ and $\quad \tilde{\mathrm{A}} \subset(\tilde{\mathrm{U}} \cup \tilde{\mathrm{V}}) \quad$ then $\min \left(\mu_{\tilde{U}}\left(c_{k}\right), \mu_{\tilde{V}}\left(c_{k}\right), \mu_{\tilde{A}}\left(c_{k}\right)\right) \neq 0$ or $(\tilde{U} \cap \tilde{V} \cap \tilde{A}) \neq \varnothing$. Pick $c \in(\tilde{U} \cap \tilde{A})$ and $d \in(\tilde{V} \cap \tilde{A})$ then $c \in \tilde{A}$ and $d \in \tilde{A}$. Since $\tilde{\mathrm{A}}$ is $\rho_{\tilde{\tau}}$-connected, there is a $\rho_{\tilde{\tau}}$-path $<\mathrm{c}=\mathrm{p}_{0}, \mathrm{p}_{1}, \ldots, \mathrm{p}_{\mathrm{n}}=\mathrm{d}>$ in $\tilde{\mathrm{A}}$ from $\mathrm{c}$ to $\mathrm{d}$. Due to $\tilde{\mathrm{A}} \subset(\tilde{\mathrm{U}} \cap \tilde{\mathrm{V}})($ assumption), then there must be a $\mathrm{k}, 1 \leq \mathrm{k} \leq \mathrm{n}$ such that $\mathrm{p}_{\mathrm{k}-1} \in \tilde{\mathrm{U}}$ or $\mu_{\tilde{\mathrm{U}}}\left(\mathrm{p}_{\mathrm{k}-\mathrm{i}}\right) \neq 0$ and $\mathrm{p}_{\mathrm{k}} \in \mathrm{p}_{\mathrm{k}} \in \tilde{\mathrm{V}}$ or $\mu_{\tilde{v}}\left(p_{k}\right) \neq 0$. Since $\left(p_{k-1}, p_{k}\right) \in \rho_{\tilde{\tau}}$, either $p_{k-1} \in S_{\tilde{\tau}}\left(p_{k}\right)$ or $\mathrm{p}_{\mathrm{k}} \in \mathrm{S}_{\tilde{\tau}}\left(\mathrm{p}_{\mathrm{k}-1}\right)$. We complete this first part of the proof assuming that the latter is the case; the proof of the alternative is strictly analogous. Since $\tilde{U}$ is an open fuzzy set which contain $p_{k-1}$, it follows from the definition of the smallest neighborhood that $S_{\tilde{\tau}}\left(p_{k-1}\right) \subset \tilde{U}$ and so $\mathrm{p}_{\mathrm{k}} \in \tilde{\mathrm{U}}$ or $\mu_{\tilde{\mathrm{U}}}\left(\mathrm{p}_{\mathrm{k}}\right) \neq 0$. Likewise, since $\mathrm{c}, \mathrm{d} \in \tilde{\mathrm{A}}$ and $<\mathrm{c}=\mathrm{p}_{0}, \mathrm{p}_{1}, \ldots, \mathrm{p}_{\mathrm{n}}=\mathrm{d}>$ is a $\rho_{\tilde{\mathrm{\tau}}}$ - path connected $\mathrm{c}$ and $\mathrm{d}$ in $\tilde{\mathrm{A}}$ then $\mathrm{p}_{\mathrm{k}} \in \tilde{\mathrm{A}}$ or $\mu_{\tilde{\mathrm{A}}}\left(\mathrm{p}_{\mathrm{k}}\right) \neq 0$. Hence, $\min \left(\mu_{\tilde{\mathrm{U}}}\left(\mathrm{c}_{\mathrm{k}}\right), \mu_{\tilde{\mathrm{V}}}\left(\mathrm{c}_{\mathrm{k}}\right), \mu_{\tilde{\mathrm{A}}}\left(\mathrm{c}_{\mathrm{k}}\right)\right) \neq 0$ or $(\tilde{\mathrm{U}} \cap \tilde{\mathrm{V}} \cap \tilde{\mathrm{A}}) \neq \varnothing$.

\section{Theorem 5}

Let $(\mathrm{V}, \tilde{\tau})$ be an Alexandroff fuzzy topological space then $V, \rho_{\tilde{\tau}}$ is a fuzzy topological digital space if and only if $\mathrm{V}$ is $\tilde{\tau}$-connected 


\section{Proof}

Since $V, \rho_{\tilde{\tau}}$ is a fuzzy topological digital space then $\mathrm{V}, \rho_{\tilde{\tau}}$ is a digital space. Therefore, $\mathrm{V}$ is $\rho_{\tilde{\tau}}$-connected (definition of digital space). By Theorem $4, \mathrm{~V}$ is $\tilde{\tau}$-connected. The same theorem implies that if this condition is satisfied, then $\mathrm{V}, \rho_{\tilde{\tau}}$ is necessarily a fuzzy topological digital space.

\section{Corollary 3}

If $\left(\mathbb{Z}^{2}, \tilde{\tau}\right)$ is a Khalimsky fuzzy topological space then $\left(\mathbb{Z}^{2}, \rho_{\tilde{\tau}}\right)$ is a digital space with $\rho_{\tilde{\tau}}$ as Khalimsky adjacency.

\section{Corollary 4}

If $\left(\mathbb{Z}^{2}, \tilde{\tau}\right)$ is a Khalimsky fuzzy topological space and $\rho_{\tilde{\tau}}$ is an adjacency induced by $\tilde{\tau}$ then $\left(\mathbb{Z}^{2}, \rho_{\tilde{\tau}}\right)$ is a fuzzy topological digital space.

\section{RESULTS AND DISCUSSION}

In this study, fEEG digitized as in (5) will be showed as a fuzzy topological digital space.

\section{Theorem 6}

$f \mathrm{EEG}$ is a Khalimsky fuzzy topological space.

\section{Proof}

Suppose $f$ EEG $=\left\{(\mathrm{x}, \mathrm{y})_{\text {Volt }} \mid \mathrm{x}, \mathrm{y} \in \mathbb{Z} ;\right.$ Volt $\left.\in \mathbb{Z}^{+}\right\}$as mentioned in (5). However $f$ EEG can be redefined as follows:

$$
\begin{aligned}
& \text { fEEG }=\left\{(\mathrm{x}, \mathrm{y})_{\text {Volt }} \mid \mathrm{x}, \mathrm{y} \in \mathbb{Z} ; \text { Volt } \in \mathbb{Z}^{+}\right\} \\
& =\left(\mathbb{Z}, \tilde{\tau}_{\mathbb{Z}}\right) \times\left(\mathbb{Z}, \tilde{\tau}_{\mathbb{Z}}\right)=\left(\mathbb{Z}^{2}, \tilde{\tau}_{\mathbb{Z}^{2}}\right)
\end{aligned}
$$

By the Theorem 3, $\left(\mathbb{Z}^{2}, \tilde{\tau}_{\mathbb{Z}^{2}}\right)$ is a Khalimsky fuzzy topological space. Therefore, $f$ EEG is a Khalimsky fuzzy topological space.

\section{Corollary 5}

$f \mathrm{EEG}$ is an Alexandroff fuzzy topological space.

\section{Proof}

By using Corollary 2 and Theorem 6.

\section{Theorem 7}

(fEEG, $\rho_{\tilde{\tau}_{\mathbb{Z}^{2}}}$ ) is a digital space

\section{Proof}

By using Corollary 3 and Theorem 6.

\section{Theorem 8}

(fEEG, $\rho_{\tilde{\tau}_{\mathbb{Z}^{2}}}$ ) is a fuzzy topological digital space.

\section{Proof}

By using Corollary 4 and Theorem 6.

\section{Theorem 9}

fEEGis $\tilde{\tau}_{\mathrm{z}^{2}}-$ connected

\section{Proof}

By using Theorem 8 and Theorem 5.

\section{CONCLUSION}

In this study, it is shown that $f$ EEG during epileptic seizure is a Khalimsky fuzzy topological space, a fuzzy topological digital space and $\tilde{\tau}_{\mathrm{z}^{2}}$ - connected.

\section{ACKNOWLEDGMENT}

The researcher gratefully acknowledges the reviewers for the constructive comments.

\section{REFERENCES}

Amari, S. and H. Nagaoka, 2000. Methods of Information Geometry. 1st Edn., American Mathematical Soc., Providence, ISBN-10: 0821843028, pp: 206.

Herman, G.T., 1998. Geometry of Digital Spaces. 1st Edn., Springer, Boston, ISBN-10: 0817638970, pp: 216.

Kiselman, C.O., 2004. Digital geometry and mathematical morphology. Uppsala University.

Kopperman, R., 2003. Topological digital topology. Discr. Geometry Comput. Imag., 2886: 1-15. DOI: 10.1007/978-3-540-39966-7_1

Melin, E., 2008. Digital Geometry and Khalimsky Spaces. 1st Edn., Department of Mathematics, Uppsala University, Uppsala, ISBN-10: 9150619837, pp: 54. 
Nazihah, A., 2009 Theoretical Foundation for Digital Space of fEEG. Universiti Teknologi Malaysia.

Nazihah, A., A. Tahir and M.I. Hussain, 2009. Topologizing the bioelectromagnetic field. Proceedings of the 5th Asian Mathematical Conference, Jun. 22-26, Kuala Lumpur.

Palaniappan, N., 2005. Fuzzy Topology. 2nd Edn., Alpha Science International, Limited, Harrow Alpha Science, ISBN-10: 1842652095, pp: 193.

Srivastava, R., S.N. Lal and A.K. Srivastava, 1981. Fuzzy hausdorff Topological Spaces. J. Math. Analysis Appli., 81: 497-506. DOI: 10.1016/0022247X(81)90078-0
Tahir, A., 2010. EEG signals during epileptic seizure as a semigroup of upper triangular matrices. Am. J. Applied Sci., 7: 540-544. DOI: 10.3844/ajassp.2010.540.544

Zakaria, F., 2008. Dynamic profiling of electroencephalographic data during seizure using fuzzy information space. $\mathrm{PhD}$ Thesis, Universiti Teknologi Malaysia. 